\section{Single photons on demand}

\author{
Barry Sanders ${ }^{1}$, Jelena Vuckovic ${ }^{2}$ and Philippe Grangier ${ }^{3}$ \\ ${ }^{1}$ Institute for Quantum Information Science, University of \\ Calgary, Alberta T2V 1G1, Canada \\ ${ }^{2}$ Ginzton Laboratory, Stanford University, California 94305- \\ 4088 USA \\ ${ }^{3}$ Institut d'Optique Théorique et Appliquée, F-91403 Orsay, \\ France
}

$\mathrm{O}$ ne hundred years since the introduction of the photon to explain the photoelectric effect, researchers are working towards creating single photons on demand. Quantum information technology is an important driver of this research effort, with single photons operating as carriers of quantum information for optical quantum computing and for quantum cryptography. Ideally, the creation of single photon on demand means providing exactly one photon, in a transform-limited wavepacket, precisely when it is required.

In reality, a variety of criteria are used to characterize a single photon source, depending on the envisioned application. Weak coherent pulses, obtained by strongly attenuating a laser beam, in order to get closer to the 'single-photon regime', provide a good comparison scale. Such pulses have a Poissonian photon number statistics, which means that they present a large vacuum component (i.e. many pulses are empty), as well as non-negligible multiphoton contributions (i.e. a fraction of the pulses contain two photons or more). By contrast, a good source of single photons should be efficient, i.e. the vacuum component (the likelihood of not getting a photon) should be small. For a given probability to get a photon, the probability to get two photons or more should be negligible compared to the Poisson value.

In addition it is desirable for applications that the source can produce a train of single photons on demand at a high repetition rate. The capability to produce single photons at or near room temperature is also desirable from a practical viewpoint. For quantum information applications, a rapid production of single photons is required to produce a large number of qubits per unit of time. Shaping of the spatio-temporal mode of the single photon is also important to provide optimal coupling efficiency with components of the quantum network, such as communication channels, quantum memory and detectors. In addition, optical quantum computation requires that all photonic qubits are identical, i.e., indistinguishable.

In recognition of the importance of single photons and the rapid advances in the field of research, the open-access journal New Journal of Physics has published a Focus Issue on "Single Photons on Demand". Representing the absolute state-of-the-art in the field, the issue features articles from some of the leading research groups working on single photons. This review of the contributions therefore provides an overview of the research area in 2004 and an inspiration of what is to come.

The advances reported in the issue include new and improved sources of single photons, characterization and applications of single photons, improved efficiency of single photon sources by interferometry and accepting the output field based on only certain photon counting outcomes, and preparing intracavity photon number states. Physical realizations of sources are quite varied, including quantum dots in pillar microcavities, parametric down-conversion, falling neutral atoms, trapped atoms in optical cavities, single defects in diamond nanocrystals, and individual molecules in a solid. The New Journal of Physics presents the latest developments in all these areas.

Moerner, from Stanford University, presents results on using single terrylene molecules in host crystals of p-terphenyl, which operate at low (liquid helium) and room temperature. Controllable emission was demonstrated for single molecules in using adiabatic rapid passage. Terrylene is a particularly interesting source as it is exhibits high photostability under continuous, intense irradiation and can operate effectively at room temperature by exploiting fast pumping into vibrational sidebands of the electronically excited state. The single-photon emission at a detected count rate of 300,000 photons per second exhibits strong antibunching, which indicates a negligible rate of multiphoton events.

Alléaume and co-workers from CNRS, the École Normale Supérieure, Laboratoire Kastler Brossel, and the Université Pierre et Marie Curie, employ the molecule DiIC18 as a room temperature single-photon source with a good photostability. They achieve strong antibunching and realized a careful analysis of intensity noise, in order to demonstrate an explicit sub-Poissonian photon statistics (negative value of the Mandel parameter) for the emitted train of single photons.

Heinrich et al, from the Max-Planck Institut für Quantenoptik, obtain single photons from neutral atoms in an optical cavity. The cooled atoms fall randomly through a high-finesse optical cavity and are driven by a periodic sequence of laser pulses. The output light is strongly antibunched, but the Poisson statistics of the number of atoms in the cavity prevents sub-Poissonian photon statistics from the source.

A collaboration by Santori et al, involving Stanford University, University of Tokyo and NTT Basic Research Laboratories of Japan, demonstrate single-photon generation with InAs quantum dots. These quantum dots are in pillar microcavities (see Fig. 1 for the schematic, scanning-electron microscope image of an actual pillar structure, and the optical excitation scheme for the quantum dot), and the effects on performance of the excitation wavelength and polarization, the collection bandwidth and polarization, are studied in detail. Measured results for efficiency of generating single photons and the purity of the states are reported, and they discuss prospects for improving these devices.

Aichele et al from Humboldt University and from ETH in Zürich also consider single photons from quantum dots. They report photoluminescence measurements from single InP and CdSe quantum dots, which produce visible photons in the visible spectral range $510-690 \mathrm{~nm}$, and characterize this photoluminescence by the autocorrelation function and via Fourier spectroscopy on several transitions in the quantum dot. In particular they observe and interpret carrier trapping and recapture in InP quantum dots, which leads to anomalies in the measured correlation functions.

In an overlapping collaboration with the work reported in the previous paragraph, Zwiller et al from ETH in Switzerland and Humboldt University discuss single quantum dots as singlequantum emitters with all the requirements to generate single photons at visible and near-infrared wavelength. They also show that single quantum dots can be used to generate non-classical states of light, from single photons to photon triplets.

A collaboration between the Universities of Stuttgart, Bremen and Würzburg, reported by Benyoucef et al, study enhanced 


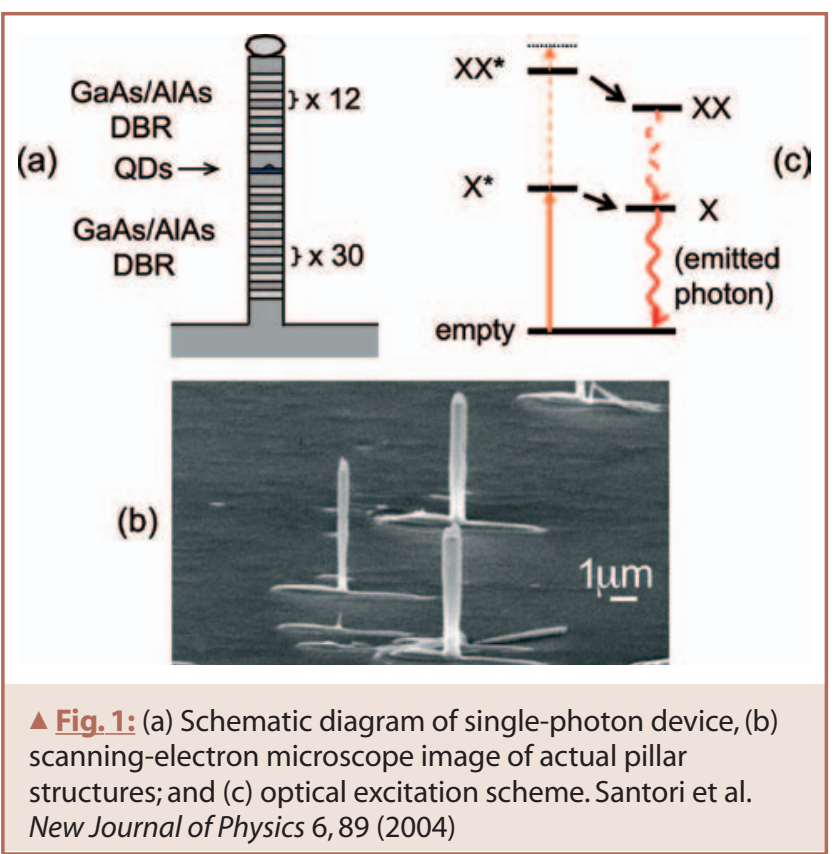

correlated photon pair emission from a pillar microcavity. They demonstrate efficient generation of triggered photon pairs and report photon cross-correlation measurements between bi-exciton and exciton decay, which reveals a bunching effect under pulsed excitation due to the cascaded nature of the emission and polarization correlation between the exciton and biexciton emission. They undertake a careful study of the emission mode structure of the pillar microcavities. The photoluminescence intensity of quantum dots in pillar microcavities yields an enhancement factor of forty in comparison with photoluminescence intensity of quantum dots in bulk semiconductors.

Brokmann et al, from the École Normale Supérieure, CNRS and Université Pierre et Marie Curie work with colloidal $\mathrm{CdSe} / \mathrm{ZnS}$ quantum dots as single-photon sources. They show that the fluorescence of colloidal CdSe/ZnS nanocrystals at room temperature exhibits perfect antibunching under continuous or pulsed excitation, and they discuss the consequences of fluorescence properties of CdSe nanocrystals on the generation of single photons. In particular, they examine the role of Auger processes in the inhibition of multiexcitonic emission and the relationship between Auger processes and the fluorescence intermittency of CdSe quantum dots.

Gaebel and collaborators from the University of Stuttgart report on generation of single photons by optical excitation of the nickel-nitrogen complex (NE8) centre in diamond, with its most striking feature being its emission bandwidth of $1.2 \mathrm{~nm}$ at room temperature. The emission wavelength of the defect is around $800 \mathrm{~nm}$, which is suitable for telecom fibres, and little background light from the diamond bulk material is detected in this spectral region. Consequently, a high contrast in antibunching measurements is achieved.

Two research groups propose a controlled single-photon source produced by a calcium ion in a high-finesse optical cavity, by exploiting Raman scattering of the pump. This scheme exploits the stable localization of the ion within the cavity, in contrast to the motion of neutral atoms. Maurer et al from the University of Innsbruck propose this scheme as a deterministic source of single photons and show that the efficiency of photon emission into the cavity mode reaches $95 \%$. Keller and collaborators at the Max-Planck-Institut für Quantenoptik and the
National Institute of Information and Communications Technology in Kobe show numerically that photons from the pump beam are Raman-scattered by the ion into the cavity mode, which subsequently emits the photon into a well-defined output channel, and present a proposal for an experiment.

Berry and his collaborators, from the Australian Centre for Quantum Computer Technology at Macquarie University, Imperial College, the Institute for Quantum Computing at the University of Waterloo, the Perimeter Institute for Theoretical Physics, and the Institute for Quantum Information Science at the University of Calgary, consider the improvement of single photon sources by mixing several photons in a train together in an interferometer, subjecting all but one of the interferometer outputs to photon counting measurements, and accepting the field from the last port only for specific photon counting data. They show that it is impossible to increase the probability for a single photon using linear optics and photodetection on fewer than four modes due to the incoherence of the inputs. If the inputs were pure-state superpositions, it would be possible to obtain a perfect single-photon output. In general, they show that a chain of beam splitters can increase the efficiency but at the expense of increasing the multiphoton component.

Thus far the emphasis has been on producing single photons on demand, but parametric down-conversion offers the prospect of heralded photons, by detecting one partner of a correlated pair of photons, and using the herald to effectively create a single photon on demand. Three research collaborations present advances on parametric down-conversion with heralded or on-demand single photons in mind.

Castelletto and her co-workers, from the Istituto Elettrotecnico Nazionale G Ferraris in Torino and the Optical Technology Division of the National Institute of Standards and Technology in Gaithersburg, present theory on the measurement of twophoton single-mode coupling efficiency in parametric down-conversion photon sources. Spontaneous parametric down-conversion creates two correlated photons from one pump photon at random times, which can serve as a source of photon pairs or, if one photon is detected, then as a heralded photon source with one photon being identified by detecting its correlated partner. In practice, the output light from the parametric down-converter is collected into a single spatial mode of an optical fibre. Castelletto et al consider two models for coupling parametric down-converters to single-mode fibres in a non-collinear configuration, and obtain different results for the two models. As a proper model is required for sophisticated experimental arrangements and improved collection efficiency, an experiment is proposed to test which model is valid.

Jeffrey et al from the University of Illinois at Urbana-Champaign present an experimentally feasible scheme to create single photons deterministically out of a non-deterministic spontaneous parametric downconversion source. They provide an analysis of efficiency for obtaining exactly one photon in the output and demonstrate a method for controlling the purity of an output photon using a partial measurement of a polarization-entangled photon pair, which is a partial implementation of remote-state preparation. The combination of these two techniques could allow on-demand preparation of single photons in arbitrary states.

The team of Fasel and co-workers from the University of Geneva, the CNRS, and the University of Nice-Sophia Antipolis report on the experimental realization and characterization of an asynchronous heralded single-photon source based on spontaneous parametric down-conversion. The $1550 \mathrm{~nm}$ photons 
are heralded as being inside a single-mode fibre with more than $60 \%$ probability, and the multi-photon emission probability is reduced by a factor of up to more than 500 compared to Poissonian light sources. These figures of merit, together with the choice of telecom wavelength for the heralded photons, are compatible with practical applications needing very efficient and robust single-photon sources.

Photodetectors are critical tools for heralded photons and also the photon efficiency enhancement proposal of Berry et al. Romestain et al of CNRS and CEA present superconducting photodetectors as an alternative to traditional photon counting systems such as photomultiplier tubes and avalanche photodiodes. They explain that different mechanisms are exploited to detect photon absorption, depending on the type of detector, whether it is a transition edge sensor, a superconducting tunnel junction, or a hot electron bolometer. Following a brief introduction to the first two types of detectors, the hot electron bolometer made with very thin superconducting $\mathrm{NbN}$ films is discussed in detail. This latter detector is promising due to its unique capability of fast single-photon detection.

Whereas the emphasis thus far has been on producing single photons, Varcoe and co-workers from the University of Sussex and the Max Planck Institute for Quantum Optics review their work on the creation and detection of arbitrary photon number states in the micromaser. Specifically they demonstrate the creation of steady-state Fock state via trapping states and the creation of dynamic Fock state via state reduction of lower state atoms. Furthermore, they analyze their experimental results regarding the creation of Fock states on demand.

Alléaume and co-workers, from Institut d'Optique and the École Normale Supérieure de Cachan describe the implementation of a BB84 quantum key distribution system using a single-photon source. The experimental scheme is depicted in Fig. 2 , and shows that each photon is transmitted through free space and classical communication is via the internet. A single nitrogen-vacancy colour centre in a diamond nanocrystal serves as the single photon source. They show that, for strong attenuation of the photons through the open night air, the use of pure singlephoton states yields a measurable advantage over systems relying on weak attenuated laser pulses.

In summary, this focus issue presents a quite complete overview of state-of-the-art single photon generation in 2004, and reports advances with sources of single photons on demand and heralded photons. The articles also describe methods for characterization, enhancement and application of these single photon sources for quantum cryptography and quantum information processing.

\section{About the authors}

Barry Sanders is Director of the Institute for Quantum Information Science at the University of Calgary and Adjunct Professor at Macquarie University, Sydney, with primary research interests in photonic implementations of quantum information.

Jelena Vuckovic is an Assistant Professor at Stanford University, with research interests in photonic crystals, quantum information, and cavity quantum electrodynamics.

Philippe Grangier is leader of the Quantum Optics group at the Institut d'Optique, Orsay, and has performed many key experiments in quantum optics, including tests of Bell's inequalities, squeezing, quantum nondemolition measurements, and more recently, in quantum information.

\section{References}

All contributions to the Focus Issue on 'Single Photons on Demand' published in New Journal of Physics (www.njp.org), are free-to-read and may be accessed directly at http://stacks.iop.org/1367-2630/6/i=1/a=E04

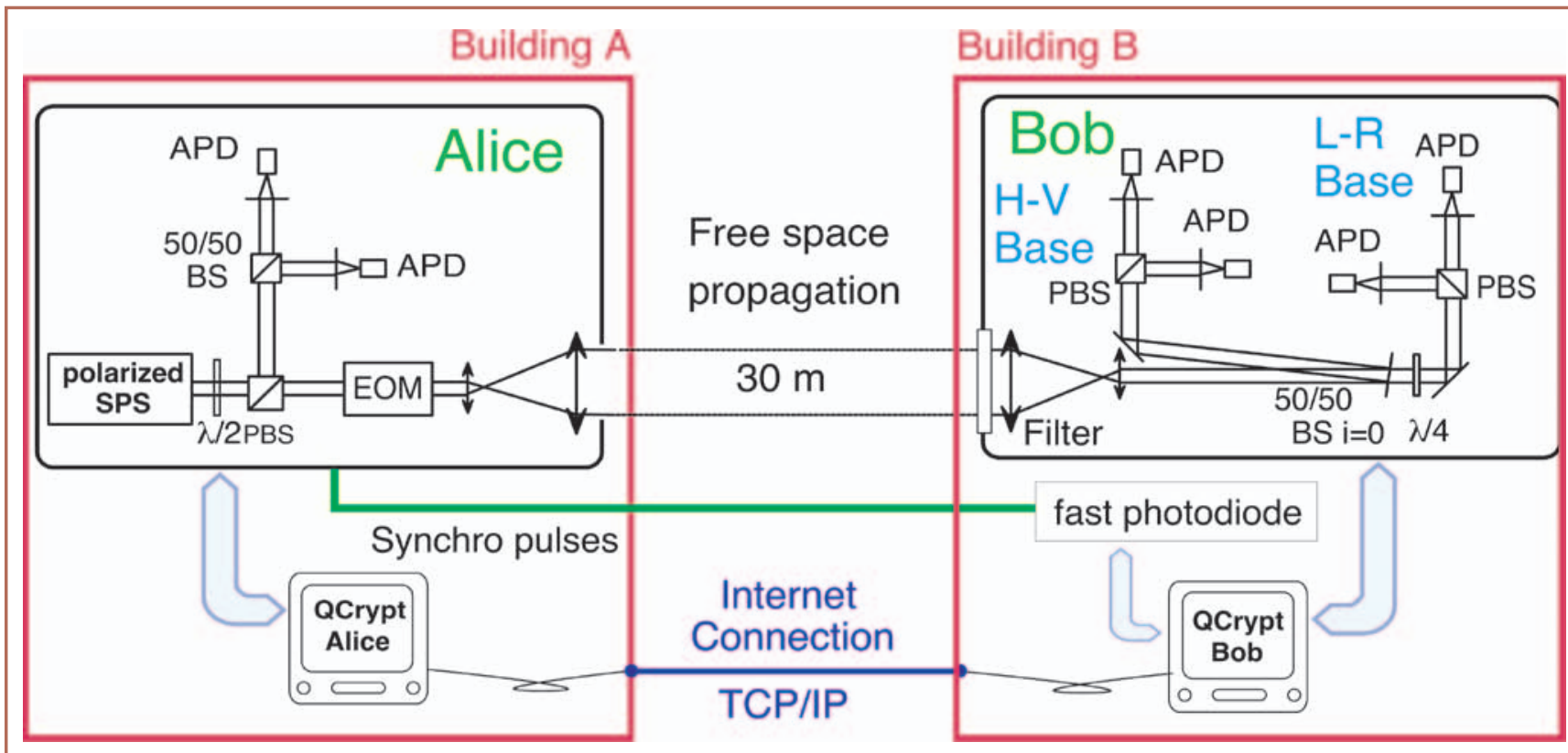

$\Delta$ Fig. 2: Experimental set-up for our quantum key distribution system based on a polarized single photon source. This system corresponds to the implementation of the BB84 protocol. It was operated at night using a free space quantum channel between Alice and Bob and the internet as the classical channel. APD, silicon avalanche photodiode; BS, beam splitter; PBS, polarizing beam splitter; EOM, electro-optical modulator; $\lambda / 2$, achromatic half-wave plate; $\lambda / 4$, achromatic quarter-wave plate. Alleaume et al. New Journal of Physics 6, $92(2004)$ 